\title{
Publisher Correction: Understanding interface stability in solid-state batteries
}

Yihan Xiao (D), Yan Wang (D), Shou-Hang Bo (D), Jae Chul Kim (1), Lincoln J. Miara and Gerbrand Ceder(D)

Nature Reviews Materials (2019) https://doi.org/10.1038/s41578-019-0157-5 Published online 09 December 2019

In this Review Article, the accept date was inadvertently omitted. It should be 24 October 2019. This error has been corrected online.

https://doi.org/10.1038/s41578-020-0191-3 I Published online 9 March 2020

๑) Springer Nature Limited 2020 